\title{
Data warehousing and knowledge discovery from sensors and streams
}

\author{
Alfredo Cuzzocrea
}

Published online: 18 August 2011

C Springer-Verlag London Limited 2011

During last years, the issue of effectively and efficiently supporting data warehousing and knowledge discovery from sensor networks, and, more generally, data stream sources, which can be reasonably intended as a meaningfully generalization of the former kind of networks, is gaining a more and more a great deal of interest from the data warehousing and knowledge discovery research community. Main research issues in this scientific field arise from the clear and well-recognized unsuitability of traditional data warehousing and knowledge discovery methodologies, techniques and algorithms in dealing with the new challenges posed by sensor network data and, more generally, data streams. Indeed, traditional approaches are meant for multi-step methodologies and techniques, and multi-scan algorithms, which cannot be straightforwardly applied to sensor network data and data streams, due to wellknown limitations such as bounded memory, online/timely data processing, need for one-pass techniques, energy consumption issues etc.

Starting from these limitations, a plethora of data warehousing and knowledge discovery methodologies, techniques and algorithms have been proposed during these last years, and, simultaneously, a more and more large number of research events have focused their attention to this leading research challenge. Following this actual trend, the special issue on "Data Warehousing and Knowledge Discovery from Sensors and Streams" of Knowledge and Information Systems puts emphasis on both theoretical and practical aspects of data warehousing and knowledge discovery from sensor network data and data streams, from foundations to theory, and from methodologies to applications.

With the aim of adequately fulfilling both theoretical and practical issues deriving from data warehousing and knowledge discovery from sensor network data and data streams, this special issue contains five papers, which have gone through two rigorous review rounds before being accepted for final inclusion. Some of the contributions of this special issue have been invited for submission as best papers from the First International Workshop on Data Warehousing and Knowledge Discovery from Sensors and Streams (DKSS 2009) held in Marina

\footnotetext{
A. Cuzzocrea $(\varangle)$

ICAR-CNR and University of Calabria, 87036 Cosenza, Italy

e-mail: kais@cems.uvm.edu
} 
Del Rey, CA, USA, during June 8-10, 2009, in conjunction with the 5th International Conference on Distributed Computing in Sensor Systems (DCOSS 2009), leaded by the Editor.

The first paper, titled "Fast and Memory Efficient Mining of High Utility Itemsets from Data Streams: with and without Negative Item Profits", by Hua-Fu Li, Hsin-Yun Huang, and Suh-Yin Lee, focuses the attention on the issue of mining utility itemsets from data steams, which has emerged as one of the most interesting research issues in data mining and knowledge discovery over data streams (as well as sensor network data). In this research, authors propose two efficient sliding window-based algorithms, called Mining High Utility Itemsets based on BITvector (MHUI-BIT) and Mining High Utility Itemsets based on TIDlist (MHUI-TID), respectively, which allow us to effectively and efficiently mine high utility itemsets from data streams. These algorithms rely on two effective representations of item information, namely Bitvector and TIDlist, respectively, and a novel lexicographical treebased summary data structure, called Tree-2HTU, whose combined application allow the efficiency of discovering high utility itemsets with positive profits as well as negative profits from data streams to be improved significantly.

The second paper, titled "One-Class Learning and Concept Summarization for Data Streams", by Xingquan Zhu, Wei Ding, Philip S. Yu, and Chengqi Zhang, introduces a novel research problem, namely concept learning and summarization for one-class data streams, which indeed turns to play a critical role in data stream mining research. According to the proposed formulation, given an input data stream set to be classified such that only one class of positive samples are labeled for training, the final goal is that of predicting whether a new instance falls into the same category as the positive examples or not. In the author's research, the main goal to be achieved with the data stream classification phase is double-fold: (i) to allow users to label instance groups, instead of single instances, as positive samples for learning; (ii) to summarize concepts labeled by users over the whole stream. With these aims in mind, authors propose a comprehensive framework, called One-Class Learning and Summarization (OCLS), which encompasses two main modules. The first module, called Vague One-Class Learning (VOCL), allows us to learn concepts from data streams by using an ensemble of classifiers with instance level and classifier level weighting strategies. The second module, called One-Class Concept Summarization (OCCS), makes use of clustering techniques and Markov models to summarize concepts labeled by users, at the cost of only one scan of the target stream.

The third paper, titled "Recommending Items in Pervasive Scenarios: Models and Experimental Analysis", by Luca Becchetti, Ugo Maria Colesanti, Alberto Marchetti-Spaccamela, and Andrea Vitaletti, proposes and experimentally assesses fully decentralized, collaborative filtering techniques, which turn to be particularly interesting in pervasive systems of small devices with limited communication and computational capabilities, like sensor networks. These collaborative filtering techniques are also extremely useful in the vest of baseline routines of complex data warehousing and knowledge discovery approaches over sensor and stream networks. In particular, in this paper, authors focus the attention on the special application scenario described by network items tagged with smart tags (such as passive RFIDs) and storing aggregate information about the visiting patterns of users that interacted with them in the past. Users access and modify information stored in smart tags transparently, by means of smart reader devices that are already available on commercial mobile phones. Smart readers make use of private information about previous behavior of users and aggregate information retrieved from smart tags in order to recommend new items that are more likely to meet user expectations.

The fourth paper, titled "Energy Conservation in Wireless Sensor Networks: A Rulebased Approach", by Suan K. Chong, Mohamed M. Gaber, Shonali Krishnaswamy, and 
Seng W. Loke, addresses the problem of energy conservation in wireless sensor networks (WSN), which is critical for a large spectrum of data warehousing and knowledge discovery algorithms over such networks. In this research context, authors propose concepts and techniques for extracting environmental information that is useful for controlling sensor operations, in order to enable sensor nodes to conserve their energy, hence prolonging the network lifetime. These concepts and techniques are implemented and embedded within the core layer of a generic sensor network framework, called Context Awareness in Sensing Environments (CASE), which specifically targets energy conservation at the network level. A subset framework of CASE, termed as CASE Compact, targets instead energy conservation at the sensor node level. The paper describes architectures and functionalities of both the two proposed frameworks and elucidates the requirements for them to operate together within a WSN for effective and efficient energy conservation.

Finally, the fifth paper, titled "Energy Saving Models for Wireless Sensor Networks", by Daniele Apiletti, Elena Baralis, and Tania Cerquitelli, again considers energy saving models on WSN, similarly to what done with the fourth paper. In this paper, authors particularly focus the attention on data acquisition and communication issues of WSN, which entail the frequent acquisition of measurements from all the sensors. Data acquisition and communication are indeed the main sources of power consumption in WSN, and, since sensors are usually battery-powered, energy saving during data collection plays a critical role with respect to the effectiveness and the efficiency of data warehousing and knowledge discovery algorithms over WSN. As a consequence, the challenge is to extend sensor lifetime by reducing communication cost and computation energy. Based on these main considerations, this paper thoroughly describes the complete design, implementation, and validation of the framework Selecting Representatives in a sensor Network (SeReNe), whose main goal consists in meaningfully discovering energy saving models over WSN in order to efficiently acquire sensor network data, given a set of historical sensor readings. To this end, SeReNe exploits different clustering algorithms to discover spatial and temporal correlations that allow the identification of sets of correlated sensors and sensor data streams, in order to select a suitable subset of representative sensors to be queried instead of querying all the sensors, hence reducing communication, computation and power costs.

The Editor would like to express his sincere gratitude to the Editor-In-Chief of Knowledge and Information Systems, Prof. Xindong Wu, for accepting his proposal of a special issue focused on data warehousing and knowledge discovery from sensors and streams, and for assisting him whenever required. The Editor would also like to thank all the reviewers who have worked within a tight schedule and whose detailed and constructive feedbacks to authors have contributed to substantial improvement in the quality of final papers. 